\title{
A stain on iron therapy
}

\section{Martin Canning}

Safety and quality pharmacist, The Prince Charles Hospital, Metro North Hospital and Health Service, Brisbane

\section{Louise Grannell}

Senior medicines information pharmacist, Alfred Health, Melbourne

\section{Keywords}

ferric carboxymaltose, injection site reaction, iron staining, laser therapy

Aust Prescr 2020;43:160-3 https://doi.org/10.18773/ austprescr.2020.051

\section{SUMMARY}

Iron staining is an unwanted and in some cases permanent adverse effect of intravenous iron administration. Cosmetically unacceptable staining may cause distress and have psychological implications for the patient.

There should be a suitable indication for parenteral iron therapy. Patients must be advised of the risk of harm and give their informed consent before receiving parenteral iron.

Strategies to minimise the risks of staining with intravenous iron include appropriate cannulation and close monitoring of the infusion. Stop the infusion if there are signs of extravasation.

Laser therapy may be a treatment option in cases of persistent discolouration due to iron staining.

\section{Introduction}

Iron deficiency is a common condition and a large contributor to anaemia.' The prevalence of iron deficiency anaemia is high in younger women and indigenous Australians. ${ }^{2}$ Treatment options to correct iron deficiency in Australia include oral and parenteral iron. ${ }^{3}$ Within the last decade the use of intravenous iron has been increasing, ${ }^{4}$ particularly in the community. This is because of newer iron salts with favourable adverse effect profiles and shorter infusion times for intravenous formulations. These include ferric carboxymaltose and ferric derisomaltose. For patients in hospital, iron polymaltose or iron sucrose can also be used.

An uncommon adverse effect of parenteral iron is skin staining (see Fig.). This is not a new phenomenon as it is a well-known adverse effect of intramuscular iron. ${ }^{5}$ Iron staining can occur with intravenous infusions if there is extravasation into the surrounding tissue. The use of intramuscular iron administration

\section{Fig. Iron stain}

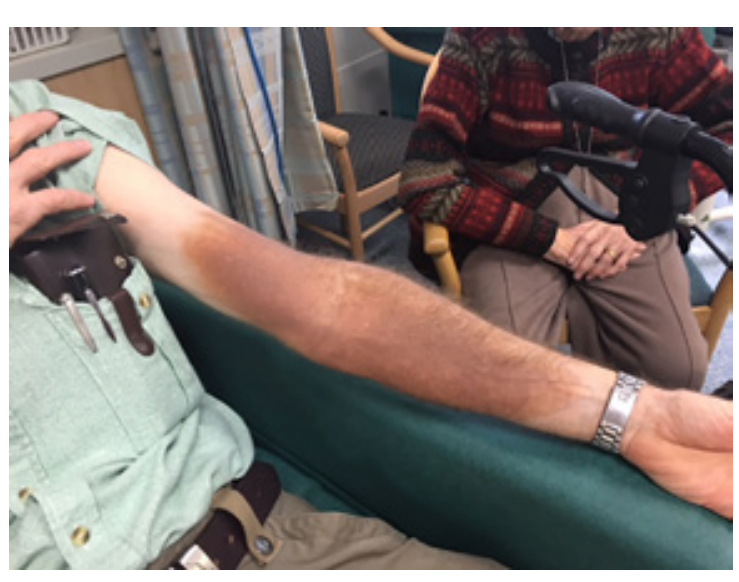

is limited in practice, ${ }^{3}$ but the injection can be given into an unexposed site. However, administration at an unexposed site is not necessarily possible when giving iron intravenously. A rise in reports of iron staining ${ }^{6-10}$ may correspond with the increasing use of intravenous iron in clinical practice. . $^{6-13}$

\section{Incidence of skin staining}

The rate of skin discolouration with intravenous iron preparations has been reported in clinical trials as $0.68 \%{ }^{14}$ to $1.3 \%{ }^{15}$ Postmarketing reports suggest the incidence may be lower and skin necrosis has not been reported. However, iron staining may be under-reported to pharmacovigilance databases. A review of the French pharmacovigilance database from 2000 to 2016 found only 51 cases of cutaneous pigmentation with iron. ${ }^{12}$

Postmarketing reports to the Therapeutic Goods Administration (TGA) Database of Adverse Event Notifications, ${ }^{16}$ from March 2014 to October 2019, included 27 cases for ferric carboxymaltose. These reports included the terms skin discolouration or hyperpigmentation, haemosiderin stain, pigmentation disorder, infusion/injection/administration site discolouration, or extravasation. The TGA data include eight cases of pigmentation disorder or skin discolouration with iron polymaltose, with the first report in 2005. There are currently no reports for ferric derisomaltose, but this adverse effect is included in the product information.

\section{Minimising harm}

Specific definitive risk factors for extravasation of intravenous iron have not been published. The principles for minimising the harm associated with intravenous iron preparations have been adapted from those applied to intramuscular iron (Box 1). They include a good infusion technique (Box 2). 


\section{Box 1 Principles for minimising the risk of intravenous iron stains}

Ensure an appropriate indication for parenteral iron Inform the patient of the risk of skin staining at the initial consultation

Ensure the correct injection site and administration technique is used

Monitor closely for signs and symptoms of extravasation

\section{Is parenteral iron indicated?}

Once iron deficiency is diagnosed, establish the cause. The decision on appropriate treatment should then consider the patient's treatment goals. This includes assessing the options for correcting the iron deficiency and their potential adverse effects.

Dietary intake, oral supplements or parenteral iron are suitable options. ${ }^{3}$

Parenteral iron is usually only indicated when oral iron therapy has failed. ${ }^{3}$ However, there are some patient cohorts who may benefit from intravenous iron without a trial of oral therapy. They include patients who have heart failure with a reduced ejection fraction, ${ }^{15}$ those undergoing haemodialysis, ${ }^{17}$ and pregnant women in their second or third trimester requiring rapid iron replenishment. ${ }^{18}$

\section{Inform patients about skin staining}

Although the incidence of iron staining appears to be relatively low, its potential irreversibility and the cosmetic impact it may have warrant discussion with patients. The Medical Board of Australia has reminded medical practitioners to advise patients about the risk so that they can give informed consent to treatment. ${ }^{19}$ Using a patient information brochure about iron staining may assist with this. The BloodSafe organisation has a useful leaflet available in English and other languages. ${ }^{20}$ When intravenous iron is indicated and patients choose to receive an infusion, it is advisable to document the content and outcome of the discussion about risks including discolouration or staining.

\section{Correct injection site and infusion technique}

The infusion sites used for intravenous therapy may influence the rate of extravasation due to the potential for vessel damage related to movement of the cannula. ${ }^{21,22} \mathrm{Administration}$ of intravenous iron via cannulation at sites of flexion (e.g. antecubital fossa, wrist) or on the back of the hand should be avoided when possible. If these sites must be used, the smallest suitable cannula size may reduce the likelihood of vessel trauma. ${ }^{22}$ Try to minimise catheter movement by securing the cannula ${ }^{21-23}$ and using an
Box 2 Infusion technique to minimise the risk of iron staining

Avoid intravenous iron administration via cannulation at sites of flexion (e.g. antecubital fossa, wrist) or on the back of the hand

The distal veins of the forearm are the preferred site Use an appropriate cannula size (20- to 24-gauge)

Secure the cannula and use an extension set to minimise catheter movement

Do not cover the injection site with a bandage Minimise the number of cannulation attempts Ensure the patency of the vein before administration. If patency is uncertain, do not administer intravenous iron Do not give infusions at night-time

Do not give infusions to patients unable to report symptoms (e.g. anaesthetised)

extension set. ${ }^{24}$ When using smaller gauge devices, it may be necessary to slow the infusion to minimise the risk of dislodgement. ${ }^{25}$

The number of attempts at cannulation should be minimised as there is an increased risk of extravasation due to multiple venous punctures. ${ }^{21,22}$ For patients who are difficult to cannulate, seek the expertise of more experienced staff. Although postponing intravenous iron therapy may inconvenience the patient, it is unlikely to result in adverse clinical outcomes. Intravenous iron infusion is rarely urgent. The patency of the cannula should be checked by giving $5-10 \mathrm{~mL}$ of sodium chloride $0.9 \%$ before the infusion. ${ }^{21}$

\section{Monitor for extravasation}

The review of cutaneous pigmentation reported to the French pharmacovigilance database suggested improvements in monitoring are necessary to detect extravasation. ${ }^{2}$ Patients who experience iron extravasation resulting in staining may describe pain, swelling, and feelings of pressure or pricking at the infusion site. ${ }^{13}$ Patients should therefore be told to notify staff of any of these symptoms (Box 3). This is an important consideration for patients who do not understand English. Administration of intravenous iron must be avoided if the patient's ability to report these symptoms is reduced (e.g. anaesthetised patients). Early cessation of the infusion may limit the amount of solution that enters the tissues and could minimise the extent of staining.

Close assessment of the cannula site during infusion is essential to enable early identification of extravasation. The site should never be covered up with a bandage. Observations of the cannula site should be timed to correspond with monitoring of the patient's other 
vital signs in accordance with local protocols for infusions. ${ }^{26}$ Giving intravenous iron infusions overnight must be avoided as it is more difficult to observe extravasation and staining in the dark.

\section{Staff training}

In order to ensure the best outcomes for patients, health professionals involved with the prescribing, administration and monitoring of intravenous iron must be adequately trained and competent. A set protocol that outlines best practice for intravenous iron administration, including cannulation, should be followed. Staff must be aware of the monitoring requirements and the symptoms of potential adverse effects.

\section{Management of iron staining}

There are no published guidelines outlining how to manage iron extravasation or skin discolouration following iron infusions. Box 4 gives the best available guidance for acute management to limit the potential for further staining. Clinical photographs should also be taken to capture the extent of the extravasation and to help with monitoring the success of subsequent treatments.

There are limited options to reverse iron staining. Topical therapies, lymphatic drainage and massages have been tried without success. ${ }^{9,13}$ The most evidence for successful reversal of iron staining is with laser therapy.

One review assessed 29 patients who had reported accidental staining from iron infusions over a nineyear period. ${ }^{13}$ Thirteen patients had laser therapy

\section{Box 3 Clinical features of iron extravasation $6-13$}

\section{Symptoms during infusion}

Pain, swelling, feeling of pressure, prickling on the injection site and immediately observable staining. Note: some patients report no pain or other symptoms during the infusion and the discolouration appears hours or days later

\section{Extent of skin discolouration}

Can be localised to around the injection site or extend along the length of the arm. May be patchy or consistent discolouration

\section{Colour changes}

Most common - light to dark brown

Less common - black, bluish, purple, grey

\section{Symptoms in the longer term}

Generally, discolouration is asymptomatic, but some patients complain of aching, changed sensitivity in the affected area or tenderness on palpation

\section{Outcome}

In many cases, iron staining is permanent. Some patients report fading of the stain over time or successful treatment with laser therapy and eight completed treatment. Regression of iron staining took an average of 5.6 laser sessions over one to two years. The type of laser is important with most evidence being for quality-switched Nd:YAG or picosecond. The patient's individual skin type may also influence the success of laser treatment. In general, laser therapy was well tolerated.

Laser therapy is available in Australia, but there may be significant financial barriers as repeated applications are required. If the patient is concerned about the staining, early referral to a dermatologist with a laser clinic specialising in quality-switched $\mathrm{Nd}$ :YAG and picosecond laser is appropriate.

\section{Review cases to improve patient safety}

When extravasation occurs, prudent review of the patient is warranted. Consider likely contributing factors, such as whether there was a suitable indication for intravenous iron, poor techniques in cannulation, the patient's own vasculature and any lack of monitoring. Report these cases to the TGA.

\section{Conclusion}

There should be a clear indication for using intravenous iron. Patients need to give informed consent for the infusion.

Iron extravasation can be cosmetically unacceptable for patients so strategies should be put in place to prevent it from occurring. These include appropriate vein selection, securing the cannula and close monitoring during the infusion. In addition, the patient should be advised to report any pain, irritation or swelling at the infusion site.

In the event of extravasation and persistent staining, repeated laser sessions over one to two years may be required. However, iron staining can be permanent. $<$

\section{Box 4 Acute management of iron extravasation}

If the patient complains of pain, swelling, soreness at the injection site or there is any obvious swelling or discolouration, stop the infusion immediately and assess the site

Disconnect the giving set

Aspirate any residual drug from the cannula

Remove the cannula

Apply a cold pack if there is swelling or soreness, however this does not appear to prevent the spread of the stain 
Conflict of interest: none declared

Acknowledgment: A thank you is extended to Carmela

Corallo, Formulary Manager at Alfred Health, for her

translation skills and review of this manuscript and

Jana Waldmann, Librarian, The Prince Charles Hospital

Library, who assisted by performing a literature search.

\section{REFERENCES}

1. Kassebaum NJ, Jasrasaria R, Naghavi M, Wulf SK, Johns N Lozano R, et al. A systematic analysis of global anemia burden from 1990 to 2010. Blood 2014;123:615-24. https://doi.org/10.1182/blood-2013-06-508325

2. Hopkins RM, Gracey MS, Hobbs RP, Spargo RM, Yates M, Thompson RC. The prevalence of hookworm infection, iron deficiency and anaemia in an aboriginal community in northwest Australia. Med J Aust 1997;166:241-4. https://doi.org/ 10.5694/j.1326-5377.1997.tb140103.x

3. Baird-Gunning J, Bromley J. Correcting iron deficiency. Aust Prescr 2016;39:193-9. https://doi.org/10.18773/ austprescr.2016.069

4. Hollands L. PBS/RPBS prescriptions for ATC4 B03AC - Iron, parenteral preparations, supplied between 1 January 2006 to 31 December 2018. Email from news@health.gov.au, 2019 Dec 3.

5. Bird S. Medication errors. Iron injections. Aust Fam Physician 2002;31:759-60.

6. Wong M, Bryson M. Extensive skin hyperpigmentation following intravenous iron infusion. $\mathrm{Br} \mathrm{J}$ Haematol 2019;184:709. https://doi.org/10.1111/bjh.15628

7. El-Zaatari MS, Hassan-Smith ZK, Reddy-Kolanu V. Extravasation and pigmentation post iron infusion. Br J Hosp Med (Lond) 2019;80:ii. https://doi.org/10.12968/ hmed.2019.80.4.i

8. Crowley CM, McMahon G, Desmond J, Imcha M. Skin staining following intravenous iron infusion. BMJ Case Rep 2019;12:e229113. https://doi.org/10.1136/bcr-2018-229113

9. Harris RE, Garrick V, Curtis L, Russell RK. Skin staining due to intravenous iron extravasation in a teenager with Crohn's disease. Arch Dis Child 2020;105:362. https://doi.org/ 10.1136/archdischild-2018-316405

10. Canning ML, Gilmore KA. Iron stain following an intravenous iron infusion. Med J Aust 2017;207:58. https://doi.org/ 10.5694/mja17.00040

11. Zuckerman MD, Greenston M. Medical image. Extraordinary extravasation. N Z Med J 2014;127:100-1.

12. Hermitte-Gandoliere A, Petitpain N, Lepelley M, Thomas L, Le Beller C, Astoul JP, et al. [Cutaneous pigmentation related to intravenous iron extravasation: analysis from the French pharmacovigilance database]. Therapie 2018;73:193-8. French. https://doi.org/10.1016/j.therap.2017.10.005

13. Eggenschwiler CD, Dummer R, Imhof L. Use of lasers for iron-induced accidental tattoos: experience at a tertiary referral center. Dermatol Surg. Epub 2019 Nov 6 https://doi.org/10.1097/DSS.0000000000002262

14. Qunibi WY, Martinez C, Smith M, Benjamin J, Mangione A, Roger SD. A randomized controlled trial comparing intravenous ferric carboxymaltose with oral iron for treatment of iron deficiency anaemia of nondialysis-dependent chronic kidney disease patients. Nephrol Dial Transplant 2011;26:1599-607. https://doi.org/ 10.1093/ndt/gfa613
15. Anker SD, Comin Colet J, Filippatos G, Willenheimer R, Dickstein K, Drexler H, et al.; FAIR-HF Trial Investigators. Ferric carboxymaltose in patients with heart failure and iron deficiency. N Engl J Med 2009;361:2436-48. https://doi.org/ 10.1056/NEJMoa0908355

16. Therapeutic Goods Administration. Database of Adverse Event Notifications (DAEN) [Internet]. Canberra: Australian Government Department of Health; 2020. www.tga.gov.au/ database-adverse-event-notifications-daen [cited 2020 Sep 1]

17. O'Lone EL, Hodson EM, Nistor I, Bolignano D, Webster AC Craig JC. Parenteral versus oral iron therapy for adults and children with chronic kidney disease. Cochrane Database Syst Rev 2019;2:CD007857. https://doi.org/10.1002/ 14651858.cd007857.pub3

18. Qassim A, Mol BW, Grivell RM, Grzeskowiak LE. Safety and efficacy of intravenous iron polymaltose, iron sucrose and ferric carboxymaltose in pregnancy: A systematic review. Aust N Z J Obstet Gynaecol 2018;58:22-39. https://doi.org/ 10.1111/ajo.12695

19. Medical Board of Australia. Case study: iron infusions what do you mean this skin stain is permanent? Update Newsletter Aug 2019. https://www.medicalboard.gov.au/ News/Newsletters/August-2019.aspx [cited 2020 Sep 1]

20. Intravenous (IV) iron infusions: information for patients, families and carers. Information Leaflet. Department of Health and Ageing, Government of South Australia. https://mytransfusion.com.au/sites/default/files/ Intravenous\%20\%28IV\%29\%20Iron\%20Infusion\%20with\%20 translations.pdf [cited 2020 Sep 1]

21. Helm RE, Klausner JD, Klemperer JD, Flint LM, Huang E. Accepted but unacceptable: peripheral IV catheter failure. J Infus Nurs 2015;38:189-203. https://doi.org/10.1097/ NAN.0000000000000100

22. Nickel B. Peripheral intravenous access: applying infusion therapy standards of practice to improve patient safety. Crit Care Nurse 2019;39:61-71. https://doi.org/10.4037/ ccn2019790

23. Helm RE. Accepted but unacceptable: peripheral IV catheter failure: 2019 follow-up. J Infus Nurs 2019:42:149-50. https://doi.org/10.1097/NAN.0000000000000324

24. Martínez JA, Piazuelo M, Almela M, Blecua P, Gallardo R, Rodríguez S, et al. Evaluation of add-on devices for the prevention of phlebitis and other complications associated with the use of peripheral catheters in hospitalised adults: a randomised controlled study. J Hosp Infect 2009;73:135-42. https://doi.org/10.1016/j.jhin.2009.06.031

25. Gill HS, Prausnitz MR. Does needle size matter? J Diabetes Sci Technol 2007;1:725-9. https://doi.org/10.1177/ 193229680700100517

26. Society of Hospital Pharmacists of Australia. Australian injectable drugs handbook. 8th ed. Melbourne: SHPA; 2020 https://www.shpa.org.au/AIDH_8th_edition [cited 2020 Sep 1]
SELF-TEST QUESTIONS

True or false?

1. Extravasation of intravenous iron leads to skin necrosis

2. The skin staining from intravenous iron usually resolves in one week

Answers on page 179 